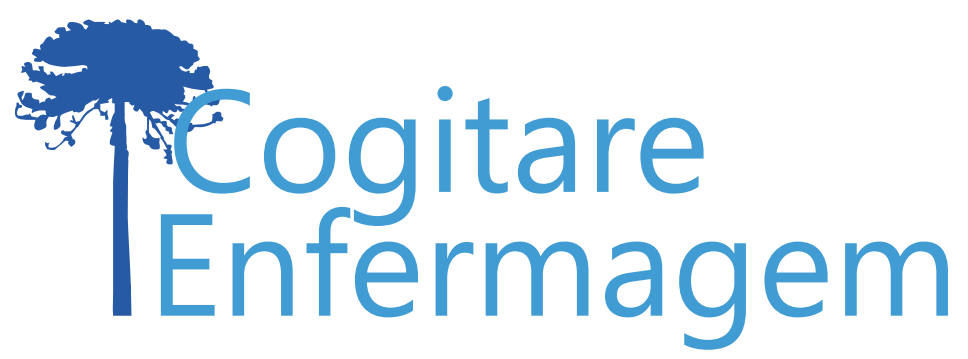

\title{
AMAMENTAÇÃO, ALIMENTAÇÃO COMPLEMENTAR E SEGURANÇA ALIMENTAR E NUTRICIONAL EM UMA REGIÃO DE FRONTEIRA
}

\author{
Aline Renata Hirano ${ }^{1}$ (1) \\ Maria Aparecida Baggio 2 \\ Rosangela Aparecida Pimenta Ferrari ${ }^{3}$ (1)
}

\section{RESUMO}

Objetivo: conhecer como a amamentação e alimentação complementar se relacionam à segurança alimentar e nutricional de crianças residentes em uma região de fronteira.

Método: pesquisa qualitativa com 12 mães e 12 profissionais de saúde de Foz do Iguaçu, no contexto da Atenção Primária à Saúde. Os dados foram coletados entre abril e outubro de 2019, por meio de entrevista semiestruturada e submetidos à Análise Temática de Conteúdo.

Resultados: foram identificadas três categorias: Aleitamento materno e alimentação complementar: direito da criança ao alimento seguro e adequado; Aleitamento materno e alimentação complementar: da orientação ao consumo; Amamentação, alimentação complementar e o contexto da Tríplice Fronteira.

Conclusão: o leite materno e a alimentação complementar saudável contemplam a segurança alimentar e nutricional. Cultura, condição financeira, trabalho materno, somados à oferta de alimentos inadequados, comprometem esse processo. Acredita-se que esse estudo possa fomentar ações e políticas públicas voltadas ao direito à alimentação infantil na região.

DESCRITORES: Leite Materno; Alimentação Complementar; Fronteira; Atenção Primária à Saúde; Segurança Alimentar e Nutricional.

\section{LA LACTANCIA MATERNA, LA ALIMENTACIÓN COMPLEMENTARIA Y LA SEGURIDAD ALIMENTARIA Y NUTRICIONAL EN UNA REGIÓN FRONTERIZA}

\section{RESUMEN:}

Objetivo: conocer como la lactancia materna y alimentación complementaria se relacionan a seguridad alimentaria y nutricional de niños que residen en una región fronteriza. Método: investigación cualitativa con 12 madres y 12 profesionales de la salud de Foz do lguaçu, en el contexto de la Atención Primaria de Salud. Los datos se recopilaron entre abril y octubre de 2019, mediante una entrevista semiestructurada y presentados para el Análisis Temática de Contenido. Resultados: se identificaron tres categorías: Lactancia materna y alimentación complementaria: el derecho del niño a una alimentación segura y apropiada; Lactancia materna y alimentación complementaria: de la orientación al consumo; Lactancia materna, alimentación complementaria y el contexto de la Triple Frontera. Conclusión: la leche materna y la alimentación complementaria saludable abarcan la seguridad alimentaria y nutricional. La cultura, la condición financiera, el trabajo materno, sumados al suministro de alimentos inadecuados, comprometen este proceso. Se cree que este estudio pueda fomentar acciones y políticas públicas orientadas al derecho a la alimentación infantil en la región.

DESCRIPTORES: Leche Humana; Alimentación Complementaria (Fenómenos Fisiológicos Nutricionales del Lactante); Frontera (Áreas Fronterizas); Atención Primaria de Salud; Seguridad Alimentaria y Nutricional. 
A segurança alimentar e nutricional faz parte dos 17 Objetivos de Desenvolvimento Sustentável do Milênio (ODS) da Agenda de 2030, visando ações globais que combatam a fome e promovam a agricultura sustentável e melhorem a nutrição de toda a população, em especial dos vulneráveis como as crianças, a partir do acesso e da disponibilidade de alimentos seguros e nutritivos ${ }^{(1)}$. Alimentação segura e adequada é o acesso permanente e frequente a alimentos em quantidade e qualidade, de acordo com as necessidades de cada pessoa, atendo-se às características sociais, culturais e biológicas, além de ser econômica e ambientalmente sustentável ${ }^{(2)}$.

No Brasil, a garantia do direito à Segurança Alimentar e Nutricional (SAN) é assegurada por meio de políticas públicas, dentre elas: a Política Nacional de Segurança Alimentar e Nutricional (PNSAN), a Política Nacional de Alimentação e Nutrição (PNAŇ) e a Política Nacional de Atenção Integral à Criança (PNAISC) ${ }^{(3-5)}$.

A prática da amamentação e da alimentação complementar saudável (ACS), condicionada à SAN, promove saúde e redução de agravos às crianças. Quando esta não é alcançada, identifica-se a Insegurança Alimentar e Nutricional (IAN), caracterizada pela disponibilidade incerta ou limitada de alimentos saudáveis e adequados e/ou condições incertas ou limitadas de acesso a alimentos em condições socialmente aceitáveis ${ }^{(6)}$.

Quando a criança é aleitada exclusivamente ao peito ou por fórmula infantil, é recomendada a introdução da alimentação complementar (AC) a partir dos seis meses, com oferta de alimentos que garantam o aporte nutricional necessário ao seu desenvolvimento ${ }^{(7)}$.

Estima-se que a promoção do aleitamento materno (AM) e da ACS possa prevenir, respectivamente, $13 \%$ e $6 \%$ de mortes de crianças em países onde as taxas de mortalidade infantil são altas ${ }^{(8)}$. No Brasil, o coeficiente de mortalidade infantil referente ao primeiro semestre do ano de 2018 era de 15,1 casos por 1000 Nascidos Vivos (NV). O estado do Paraná e o município de Foz do lguaçu, local do estudo, no mesmo período, apresentaram, respectivamente, 10,9 e 13 casos por 1000 NV. Apesar dessas taxas serem categorizadas como baixas, segundo classificação da Organização Mundial de Saúde (OMS), elas permanecem acima da meta estipulada pelo mesmo órgão, cujo valor seria abaixo de 10 mortes por $1000 \mathrm{NV}^{(9-10)}$.

Foz do Iguaçu-PR faz parte da Tríplice Fronteira, composta por Brasil, Argentina e Paraguai. Em região de fronteira, os limites territoriais podem favorecer a separação e a segregação e ao mesmo tempo coexistir a possibilidade de integração entre os povos, com consequente influência cultural e social nos aspectos do AM e da $A_{C S} S^{(11-13)}$.

Conforme o exposto, o estudo tem como objetivo conhecer como a amamentação e alimentação complementar se relacionam à segurança alimentar e nutricional de crianças residentes em uma região de fronteira.

\section{MÉTODO}

Pesquisa qualitativa derivada do projeto "Rede Mãe Paranaense na perspectiva da usuária: o cuidado da mulher no pré-natal, parto, puerpério e da criança". Foram critérios de inclusão: ser mãe de criança de oito a 24 meses de idade, que tenha sido ou ainda seja aleitada em seio materno, em AC e em realização de puericultura na Atenção Primária à Saúde (APS); ser profissional da APS (médicos, enfermeiras, nutricionistas) atuante em Unidades Básicas de Saúde (UBS), na realização de puericultura há mais de um ano ou 
envolvido na orientação de AM e AC. Ambos com interesse e disponibilidade para participar da pesquisa. Critério de exclusão: possuir menos de 18 anos e ausência de domínio da língua portuguesa, não estando apto para compreender e responder à entrevista.

Foram participantes do estudo 12 mães, sendo sete delas brasileiras e cinco estrangeiras residentes no Brasil (paraguaia, chilena, venezuelana, haitiana, peruana) e 12 profissionais da APS: dois médicos, duas nutricionistas e oito enfermeiras.

A pesquisa foi realizada no âmbito da APS, em 10 UBS dos cinco distritos sanitários do município de Foz do Iguaçu-PR, localizado na Tríplice Fronteira, compartilhado com o Paraguai e a Argentina. As ỦBS foram escolhidas intencionalmente para contemplar as que realizassem puericultura a crianças de famílias estrangeiras e brasiguaias (brasileiros residentes no Paraguai).

Os dados foram coletados de março a outubro de 2019, por meio de entrevista individual, semiestruturada e gravada, com duração média de 50 minutos. As perguntas realizadas buscaram contemplar aspectos biológicos, sociais e culturais do processo de AM e alimentação complementar, a fim de relacionar à SAN e ao contexto vivenciado na fronteira. A coleta foi encerrada pelo fenômeno de saturação de dados, que não se pauta na quantidade de entrevistas, mas na profundidade das informações obtidas que tornem possível explicar o fenômeno estudado(14).

Os dados foram analisados por meio da análise temática de conteúdo. Na pré-análise, foi realizada leitura extenuante e organização dos dados; na exploração do material, foram identificadas as unidades de sentido, dando origem às categorias e subcategorias; no tratamento dos dados obtidos e interpretação, realizados a partir da organização das categorias, foram interpretados os dados de acordo com o objetivo estabelecido(15).

O anonimato foi garantido por meio da letra " $\mathrm{M}$ ", no caso das mães, das letras "Me" no caso dos médicos e pela inicial das demais profissões, seguido do número referente à ordem das entrevistas [E1, N1, M1 (mãe), Me1 (médico)]. A pesquisa foi aprovada pelo Comitê de Ética em Pesquisa com Seres Humanos da Universidade Estadual de Londrina sob número 2.053.304.

\section{RESULTADOS}

Três categorias configuram os resultados e discorrem, quanto ao $A M$ e AC, acerca do direito da criança ao alimento seguro e adequado, da orientação profissional em saúde à prática pelas mães, bem como aspectos de uma região de Tríplice Fronteira.

\section{Aleitamento materno e alimentação complementar: direito da criança ao alimento seguro e adequado}

Dentre os profissionais participantes, médicos e enfermeiros apresentaram desconhecimento ou conhecimento superficial a respeito do que se considera um alimento seguro e adequado para a SAN da criança.

[...] Não tenho certeza. É mais para especialista nutricionista. (E2)

[...] Não sei. Gostaria de ter essa informação. (Me1)

No entanto, esses profissionais, assim como os demais participantes, afirmaram que - LM, juntamente com a ACS, possui inúmeros benefícios, associados à sua composição, à possibilidade de acesso (mesmo diante de condições financeiras desfavoráveis) e ao 
vínculo mãe-bebê, que culminam numa melhor imunidade, desenvolvimento cognitivo, neuropsicomotor, respiratório e emocional da criança. Além disso, previnem o déficit ou excesso de peso. Esses pontos positivos se estendem ao desempenho escolar e laboral futuro.

[...] recebendo nutrientes que ela precisa, tá recebendo carinho que ela também precisa quando tá assustada. Quando chorava [...] eu dava leite pra ela e parece que acalmava, sentia uma sensação de estar entregando uma coisa que era para o bem-estar dela. (M6)

[...] amamentação, alimentação complementar correta vai influenciar na saúde dela [criança] no geral, desde pequena, nos estudos, na fase adulta, no trabalho e [...] vai despender menos recursos do sistema de saúde [...]. (N1)

[...] a questão da amamentação é tão importante, não só para o desenvolvimento neuropsicomotor [...] também a cognitividade, a criança fica mais inteligente. (E2)

[...] tem menos complicações, principalmente respiratórias. [...] (N2)

[...] evitar doenças futuras, [...] obesidade, [...] baixo peso. (E1)

O peito permitiu que ele comesse, como não tínhamos recurso, esse era a única coisa que tínhamos para alimentá-lo. (M12)

$O$ retorno às atividades laborais das mães favoreceu o desmame precoce e a $A C$ inadequada, principalmente quando antes dos seis meses de vida da criança e sem a adesão à ordenha.

A médica falou que ele deu uma emagrecidinha [...]. Comecei a trabalhar [...], daí tem hora que ele fica sem comer [...] Ter voltado a trabalhar foi uma dificuldade. (M3)

O trabalho é o motivo principal de antecipação da retirada do leite [materno] [...]. (E6)

São pouquíssimas mães que fazem essa prática da ordenha. (N1)

$\mathrm{Na}$ opinião dos profissionais, ter outra fonte de leite para as crianças, por meio do Programa Leite das Crianças (PLC), pode favorecer o desmame precoce. Por outro lado, se ao receber o leite do PLC, a mãe mantiver o LM, este pode incrementar a rotina alimentar da família e aumentar a segurança alimentar da criança.

[...] elas [mães] acreditam que seja bom, porque se já tem esse leite, então com seis meses é melhor tirar do peito e já dar o "leite do governo". (N1)

[...] a criança até nem tomava o leite porque ainda mamava no peito, mas daí ajudava os outros irmãos e a família [...] para ela comprar um outro alimento. (E1)

\section{Aleitamento materno e alimentação complementar: da orientação ao consumo}

Os profissionais contraindicam a adição de açúcar aos alimentos e a oferta de produtos industrializados à criança. Contudo, a oferta desses produtos é comum e está relacionada à crença em relação ao peso da criança, preço, praticidade, palatabilidade desses produtos e, também, descaso ou hábito familiar:

[...] ao invés de você comprar um suco artificial, você pode oferecer uma fruta. [...] é um tempo que a pessoa não quer gastar naquele momento com a criança [...]. É por desleixo, descaso. (E4)

Todas elas [mamadeiras] com as gotinhas de açúcar dele. Final de semana ele toma um pouquinho de refrigerante, o achocolatado, o iogurte, o leite fermentado. (M2)

[...] as besteiras são muito fáceis, muito acessíveis, muito baratas [...]. (Me2) 
Foi identificada a monotonia alimentar na rotina das crianças, cuja ocorrência está associada à dificuldade financeira, falta de conhecimento da mãe, hábito familiar, praticidade e seletividade infantil.

[...] a gente fala: "[...] você precisa colocar nem que seja pouquinho, não precisa ser uma carne cara $[. . .]^{\prime \prime}$. Mas, às vezes, ela não tem isso [...] ela vai dar o que vai matar a fome. (E3)

[...] Não é sempre que nós podemos comprar [frutas e verduras]. E a carne também é o mais difícil. (M3)

[...] elas pegam umas coisas mais fáceis [...], eles sempre querem dar a mesma coisa. (E6)

[...] ela está com uma comida muito, assim, similar todo dia [...] talvez não tenha os nutrientes suficientes para desenvolver-se bem [...]. (M6)

Dentre as ações realizadas para a garantia do direito ao alimento seguro e adequado à criança, os profissionais orientavam sobre os benefícios do AM às mães desde o pré-natal e reiteravam nas consultas de puericultura bem como nas visitas domiciliares; já as mães priorizavam gastos com alimentação saudável do filho em detrimento a outros consumos.

Eu acredito que a função é fazer essa propaganda [...], do benefício do aleitamento materno [...] A gente trabalha no pré-natal, na puericultura, nas visitas. (E6)

[...] priorizo antes que a roupa, antes que sair ao shopping né. Então, priorizo primeiro quanto que eu vou gastar nas frutas, verduras, carnes, leite, na hora de fazer contas, para primeiro estar sua alimentação. (M12)

\section{Amamentação, alimentação complementar e o contexto da Tríplice Fronteira}

Quanto às mães paraguaias ou brasiguaias (brasileiras que residem no Paraguai), foi possível notar uma tendência ao desmame precoce bem como à introdução prévia de alimentos, provavelmente condições relacionadas com a cultura e condição financeira dos pais.

É que lá no Paraguai tem o costume de tirar muito cedo a criança do peito [...]. (M4)

[...] eles [paraguaios e brasiguaios] querem começar a dar comida muito cedo também. (E7)

Mães que residem no Brasil e trabalham no Paraguai (fronteira com o Brasil) também apresentam dificuldades para manter a amamentação, a priori vinculada à distância.

[...] elas têm essa dificuldade, porque elas trabalham no Paraguai. Não tem essa possiblidade de sair meia hora para amamentar [...] porque a gente sabe a dificuldade de vir de lá pra cá. (E7)

O diálogo, o entendimento e o respeito dos profissionais de saúde às diferentes culturas apresentam-se como norteadores ao atendimento às mães brasileiras ou estrangeiras - assim como a todos os usuários - quanto à prática da amamentação e alimentação complementar.

Ele [profissional] deve estar aberto às outras culturas [...] ao ouvir, sem desprezar aquela informação e [...] tentar passar a orientação correta. (E5) 
Para que o direito à alimentação infantil segura e adequada seja protegido pela APS, é recomendada a educação continuada de seus profissionais para qualificação de mão de obra quanto aos aspectos legais e técnicos sobre a SAN, além do manejo com os usuários por meio da promoção de ações de alimentação e nutrição(16).

Os benefícios acerca do LM e da ACS ao desenvolvimento biopsicossocial da criança corroboram com a literatura ${ }^{(17-21)}$. Contudo, apesar da responsabilidade intrínseca à família e aos profissionais de saúde para a garantia do direito a esses benefícios à criança, são requeridas melhores condições de vida e de trabalho, como empregos que favoreçam a manutenção do LM pelas mães, acesso à ACS e profissionais com qualificação e atuação engajada com SAN ${ }^{(16)}$.

O LM se destaca como um dos principais meios contra a IAN por não dispender gastos, ser nutricionalmente completo e atender de forma equitativa as necessidades individuais de cada criança. Contudo, menores taxas de amamentação são influenciadas pela ausência paterna, exposição ao cigarro, mais de três filhos, baixa escolaridade materna e a mãe trabalhar fora ${ }^{(22)}$. Este último confirmado neste estudo.

A mãe trabalhar fora de casa possui associação com o desmame precoce, podendo representar 26 vezes menos chance de manter o aleitamento materno exclusivo (AME) quando comparado à que não trabalha ${ }^{(23)}$. Diante disso, a licença maternidade de seis meses é essencial para a oferta do LM, conforme recomendação do MS e da OMS ${ }^{(7,24)}$. No entanto, este estudo mostra discrepância entre a recomendação desses órgãos e o direito de licença das mães, que não contempla o período de AME.

Uma possiblidade de manter a amamentação da mãe trabalhadora é a ordenha, incentivada pelo $\mathrm{MS}^{(7)}$. Contudo, a dor ao retirar o leite, julgar ser insuficiente a quantidade ordenhada, não dispor de tempo e local adequado para efetuar o procedimento e armazenamento do leite e, ainda, sentir-se constrangida frente aos demais colegas pode prejudicar a adesão das mulheres ${ }^{(25)}$. Além disso, os dados evidenciam descrença dos profissionais da APS para incentivar a prática.

Na ocorrência do desmame e impossibilidade da ordenha, a opção mais adequada para a criança é a fórmula infantil. Devido ao custo desse produto, o leite de vaca tende a ser a escolha de muitas famílias. Nesse sentido, o governo estadual, com a finalidade de garantir a SAN infantil, estabeleceu o PLC, destinado a crianças de seis a trinta e seis meses, que tenham renda familiar per capita inferior a meio salário mínimo ${ }^{(26)}$. No entanto, sinaliza-se necessidade de reestruturação desse programa a fim de que o recebimento desse leite não se configure como um substituto do LM ou da fórmula infantil, quando esta se faz necessária(27).

Outro fator recorrente à IAN é a oferta de ultraprocessados às crianças. Neste estudo, ele está interligado à renda familiar, desmame e introdução alimentar precoce, além da praticidade e palatabilidade. Apesar das orientações dos profissionais quanto à não oferta, a adesão dos pais nem sempre acontece. Isso alerta sobre a importância do olhar atento pelo profissional e de ações de educação em saúde com vistas à promoção do AME e da introdução da $A C$ de forma adequada ${ }^{(19)}$.

Sobre a monotonia alimentar da criança, é importante o contato com variedades de alimentos durante a infância, associado à vinculação entre gosto, cheiro, cor e textura dos alimentos, a partir de uma construção diária mediada pela família e orientada pelos profissionais de saúde da APS(7).

Experiências diferentes sobre o AM relacionadas à mesma nacionalidade, como das mães paraguaias, possivelmente tem relação com a realidade de cada mãe, uma vez que a amamentação é influenciada por diversos fatores (alguns já citados neste estudo), além dos culturais ${ }^{(23,28)}$. Diante disso, a competência cultural deve ser requisito a ser alcançado por toda a equipe de saúde, substancialmente em uma região de fronteira, e pode ser desenvolvida por meio de formação continuada. A partir da reflexão, discussão e prática, 
pode ser consolidado um atendimento baseado no respeito, diálogo ${ }^{(29)}$ e nas necessidades das mães, respeitando seus saberes culturais.

Sendo a SAN um dos ODM da Agenda $2030^{(1)}$ e a criança e o imigrante estarem entre o público mais vulnerável à IAN(30), este estudo demonstra a importância de pensar o AM e a AC como direito da criança em região de fronteira, que deve ser garantido, além das escolhas da família ou das orientações dos profissionais. Existe a necessidade de ambos terem condição de exercerem tais atribuições, o que nem sempre é realidade. Contudo, independente desta situação, as diretrizes das políticas públicas apresentadas devem ser conhecidas e seguidas de acordo com as possibilidades, reivindicando à gestão pública, por meio do controle social, para que ela seja cumprida.

Esta pesquisa apresentou como limitações o fato de as mães entrevistadas estarem acompanhadas dos filhos, com necessidade de atenção às demandas da criança, o que pode ter interferido na profundidade das informações fornecidas.

\section{CONSIDERAÇÕES FINAIS}

O LM e a ACS se confirmaram como importantes para a garantia da SAN. Contudo, a condição financeira das famílias, seguida pelo retorno ao trabalho antes dos 6 meses (com consequente desmame precoce), a oferta de produtos ultraprocessados às crianças, a falta de variedade na rotina alimentar infantil, entre outros, comprometem a sua garantia.

No que se refere à região de fronteira, os resultados expressam peculiaridades, como a influência cultural sobre o desmame ou sobre a introdução precoce de alimentos, a mulher trabalhadora que deixa de amamentar devido à dificuldade de deslocamento entre fronteiras e, ainda, a atuação do profissional de saúde junto a mães de diferentes nacionalidades na APS.

Ações realizadas pelos profissionais de saúde e mães, priorizando a alimentação da criança e os benefícios do LM e da ACS, são exemplos consolidados para garantir a SAN infantil. Soma-se a isso a importância do respeito e diálogo no atendimento às crianças estrangeiras e brasiguaias quanto às orientações referentes à alimentação adequada a esse público, potencializada pelo fato dela e do imigrante serem um grupo de risco à ocorrência de IAN.

Indica-se ampliação de estudos e a integração de políticas públicas interfronteiras a partir da realidade e das demandas próprias desse público para garantia do direito à amamentação e à ACS, tendo participação de gestores, profissionais, mães/famílias. Neste sentido, este estudo pode fomentar ações e políticas públicas voltadas à região de fronteira.

\section{AGRADECIMENTOS}

Estudo com financiamento: Chamada Universal/CNPq 01/2016 - Faixa A. Processo n. 407508/2016-3. 
1. Organização das Nações Unidas Brasil. Objetivos de desenvolvimento sustentável. [Internet]. 2016 [acesso em 08 out 2019]. Disponível em: https://nacoesunidas.org/pos2015/.

2. Brasil. Lei n. 11.346, de 15 de setembro de 2006. Cria o Sistema Nacional de Segurança Alimentar e Nutricional. SISAN com vistas em assegurar o direito humano à alimentação adequada e dá outras providências. Diário Oficial da União, [Internet]. 18 set 2006 [acesso em 08 out 2019]. Disponível em: http://www4.planalto.gov.br/consea/conferencia/documentos/lei-de-seguranca-alimentar-e-nutricional.

3. Ministério da Saúde (BR). Decreto n. 7.272, de 25 de agosto de 2010: regulamenta a Lei n. 11.346, de 15 de setembro de 2006, a qual institui a Política Nacional de Segurança Alimentar e Nutricional - PNSAN, estabelece os parâmetros para a elaboração do Plano Nacional de Segurança Alimentar e Nutricional, e dá outras providências. Diário Oficial da União, [Internet]. 26 ago 2010 [acesso em 08 out 2010]. Disponível em: http://www.planalto.gov.br/ccivil 03/ Ato2007-2010/2010/Decreto/D7272.htm.

4. Ministério da Saúde (BR). Secretaria de Atenção à Saúde. Departamento de Atenção Básica. Política Nacional de Alimentação e Nutrição. Brasília: Ministério da Saúde; 2013 [acesso em 01 mar 2018]. Disponível em: http://bvsms.saude.gov.br/bvs/publicacoes/politica nacional_alimentacao nutricao.pdf.

5. Ministério da Saúde. Portaria $n^{\circ} 1.130$, de 5 de agosto de 2015. Institui a Política Nacional de Saúde da Criança (PNAISC) no âmbito do Sistema Único de Saúde (SUS). Brasília, DF: Ministério da Saúde, 2015 [acesso em 21 jun 2018]. Disponível em: http://bvsms.saude.gov.br/bvs/saudelegis/gm/2015/ prt1130 $05 \quad 08$ 2015.html.

6. Starr MC. Fisher K, Thompson K, Thurber-Smith K, Hingorani S. A pilot investigation of food insecurity among children seen in an outpatient pediatric nephrology clinic. Preventive Medicine Reports [Internet]. 2018 [acesso em 10 dez 2018]; 10. Disponível em: https://doi.org/10.1016/j.pmedr.2018.02.019.

7. Ministério da Saúde (BR). Secretaria de Atenção Primária à Saúde. Departamento de Promoção da Saúde. Dez passos para uma alimentação saudável: guia alimentar para crianças brasileiras menores de dois anos: um guia para o profissional da saúde na atenção básica. Brasília: Ministério da Saúde; 2019 [acesso em 10 nov 2019]. Disponível em: http://189.28.128.100/dab/docs/portaldab/publicacoes/guia da crianca 2019.pdf.

8. Jones G, Steketee RW, Black RE, Bhutta ZA, Morris SS. How many child deaths can we prevent this year? The Lancet [Internet]. 2003 [acesso em 10 jun 2018]; 362(9377). Disponível em: https://doi. org/10.1016/S0140-6736(03)13811-1.

9. World Health Organization (WHO). World health statistics 2018: monitoring health for the SDGs, sustainable development goals. Geneva: WHO; 2018 [acesso em 01 dez 2018]. Disponível em: https:// apps.who.int/iris/bitstream/handle/10665/272596/9789241565585-eng.pdf?ua=1\&ua=1.

10. Secretaria de Estado da Saúde do Paraná (SESA). Superintendência de Vigilância em Saúde - SVS. Centro de Epidemiologia - CEPI. Divisão de Informações Epidemiológicas -DVIEP. Mortalidade infantil. 2018. [acesso em 02 nov 2018]. Disponível em: http://www.saude.pr.gov.br/arquivos/File/Mortalida Materna e Infantil 1807 2018.pdf.

11. Ferrari, M. As noções de fronteira em geografia. Revista Perspectiva Geográfica [Internet]. 2014 [acesso em 10 dez 2018]; 9(10). Disponível em: http://e-revista.unioeste.br/index.php/pgeografica/article/ view/10161.

12. Silva ML da, Tourinho HLZ. Território, territorialidade e fronteira: o problema dos limites municipais e seus desdobramentos em Belém/PA. Urbe. Rev. Bras. Gest. Urbana [Internet]. 2016 [acesso em 10 jun 2018]; 9(1). Disponível em: https://doi.org/10.1590/2175-3369.009.001.ao09.

13. Ruiz-Holguin R. Breastfeeding: a view from the border. Breastfeeding Medicine [Internet]. 2016 [acesso em 02 jan 2020]; 11(8). Disponível em: https://doi.org/10.1089/bfm.2016.0109.

14. Minayo MC de S. Amostragem e saturação em pesquisa qualitativa: consensos e controvérsias. Revista Pesquisa Qualitativa [Internet]. 2017 [acesso em 01 mar 2019]; 5(7). Disponível em: https://editora. sepq.org.br/index.php/rpq/article/view/82/59. 
15. Minayo MC de S (Org.). Pesquisa social: teoria, método e criatividade. 34. ed. rev. atual. Petrópolis: Vozes; 2015.

16. Botelho FC, França Junior I. Como a atenção primária à saúde pode fortalecer a alimentação adequada enquanto direito na América Latina? Rev Panam Salud Publica [Internet]. 2018 [acesso em 10 jan 2020]; 42. Disponível em: https://doi.org/10.26633/RPSP.2018.159.

17. Victora CG, Bahl R, Barros AJD, França GVA, Horton S, Krasevec J, et al. Breastfeeding in the 21st century: epidemiology, mechanisms, and lifelong effect. The Lancet [Internet]. 2016 [acesso em 01 abr 2020]; 387(10017). Disponível em: https://www.thelancet.com/journals/lancet/article/PIIS01406736(15)01024-7/fulltext.

18. Simon VGN, Souza JMP de, Souza SB de. Aleitamento materno, alimentação complementar, sobrepeso e obesidade em pré-escolares. Rev. Saúde Pública [Internet]. 2009 [acesso em 09 dez 2019]; 43(1). Disponível em: https://doi.org/10.1590/S0034-89102009000100008.

19. Giesta JM, Zoche E, Corrêa R da S, Bosa VL. Fatores associados à introdução precoce de alimentos ultraprocessados na alimentação de crianças menores de dois anos. Ciênc. saúde coletiva [Internet]. 2019 [acesso em 10 jun 2020]; 24(7). Disponível em: https://doi.org/10.1590/1413-81232018247.24162017.

20. Krol KM, Grossmann T. Psychological effects of breastfeeding on children and mothers.

Bundesgesundheitsbl Gesundheitsforschung Gesundheitsschutz [Internet]. 2018 [acesso em 02 nov 2019]; 61(8). Disponível em: https://doi.org/10.1007/s00103-018-2769-0.

21. Rollins NC, Bhandari N, Hajeebhoy N, Horton S, Lutter CK, Martines JC, et al. Why invest, and what it will take to improve breastfeeding practices? The Lancet [Internet] 2016 [acesso em 01 nov 2019]; 387(10017). Disponível em: https://doi.org/10.1016/S0140-6736(15)01044-2.

22. Venu I, Heveul M van den, Wong JP, Borkhoff CM, Moodie RG, Ford-Jones EL, et al. The breastfeeding paradox: relevance for household food insecurity. Pediatrics \& Child Health [Internet]. 2017 [acesso em 10 out 2019]; 22(4). Disponível em: https://doi.org/10.1093/pch/pxx067.

23. Tadesse F, Alemayehu Y, Shine S, Asresahegn H, Tadesse T. Exclusive breastfeeding and maternal employment among mothers of infants from three to five months old in the Fafan zone, Somali regional state of Ethiopia: a comparative cross-sectional study. BMC Public Health [Internet]. 2019 [acesso em 10 jan 2020]; 19(1015). Disponível em: https://doi.org/10.1186/s12889-019-7345-5.

24. World Health Organization (WHO). Guideline: protecting, promoting and supporting breastfeeding in facilities providing maternity and newborn services. Geneva: WHO; 2017 [acesso em 20 jan 2020]. Disponível em: https://www.who.int/nutrition/publications/guidelines/breastfeeding-facilities-maternitynewborn/en/.

25. Ismail TAT, Sulaiman Z, Jalil R, Muda WMW, Man NNN. Breast milk expression among formally employed women in urban and rural Malaysia: a qualitative study. Int Breastfeed J. [Internet]. 2012 [acesso em 10 nov 2019]; 7(11). Disponível em: https://10.1186/1746-4358-7-11.

26. Paraná. Decreto n. 3000 de 07 dezembro de 2015: regulamenta o Programa Leite das Crianças, instituído pela Lei n. 16.385, de 25 de janeiro de 2010, Curitiba, PR [acesso em 02 nov 2019]. Disponível em: http://www.leitedascriancas.pr.gov.br/modules/conteudo/conteudo.php?conteudo=18.

27. Saldan PC, Venancio SI, Saldiva SRDM, Vieira DG, Mello DF de. Consumo de leites em menores de um ano de idade e variáveis associadas ao consumo de leite não materno. Rev. paul. Pediatr. [Internet] 2017 [acesso em 08 dez 2019]; 35(4). Disponível em: https://doi.org/10.1590/1984-0462/;2017;35;4;00004.

28. Scott A, Shreve M, Ayers B, McElfish PA. Breast-feeding perceptions, beliefs and experiences of Marshallese migrants: an exploratory study. Public Health Nutr [Internet]. 2016 [acesso em 01 nov 2019]; 19(16). Disponível em: https://doi.org/10.1017/S1368980016001221.

29. Kaihlanen AM, Hietapakka L, Heponiemi T. Increasing cultural awareness: qualitative study of nurses' perceptions about cultural competence training. BMC Nurs [Internet]. 2019 [acesso em 10 jan 2020]; 18(38). Disponível em: https://doi.org/10.1186/s12912-019-0363-x. 
30. Guerra LD da S, Cervato-Mancuso AM, Bezerra ACD. Alimentação: um direito humano em disputa - focos temáticos para compreensão e atuação em segurança alimentar e nutricional. Ciênc. saúde coletiva [Internet]. 2019 [acesso em 10 jan 2020]; 24(9). Disponível em: https://doi.org/10.1590/141381232018249.20302017.

COMO REFERENCIAR ESTE ARTIGO:

Hirano AR, Baggio MA, Ferrari RAP. Amamentação, alimentação complementar e segurança alimentar e nutricional em uma região de fronteira. Cogitare enferm. [Internet]. 2021 [acesso em "colocar data de acesso, dia, mês abreviado e ano"]; 26. Disponível em: http://dx.doi.org/10.5380/ce.v26i0.72739.

*Artigo extraído da dissertação de mestrado "Amamentação e alimentação complementar de crianças em região de fronteira e sua interface com a segurança alimentar e nutricional". Universidade Estadual do Oeste do Paraná, 2020.

Recebido em: 08/04/2020

Aprovado em: 14/09/2020

Editora associada: Tatiane Herreira Trigueiro

Autor Correspondente:

Aline Renata Hirano

Universidade Estadual do Oeste do Paraná - Foz do Iguaçu, PR, Brasil

E-mail: alinerenatahirano@gmail.com

Contribuição dos autores:

Contribuições substanciais para a concepção ou desenho do estudo; ou a aquisição, análise ou interpretação de dados do estudo - ARH, MAB

Elaboração e revisão crítica do conteúdo intelectual do estudo - ARH, MAB

Aprovação da versão final do estudo a ser publicado - ARH, MAB, RAPF

Responsável por todos os aspectos do estudo, assegurando as questões de precisão ou integridade de qualquer parte do estudo - ARH

Copyright $\odot 2021$ Este é um artigo em acesso aberto distribuído nos termos da Licença Creative Commons Atribuição, que permite o uso irrestrito, a distribuição e reprodução em qualquer meio desde que o artigo original seja devidamente citado. 